\title{
LE PARADIGME ACTIONNEL EN PHILOSOPHIE DU LANGAGE
}

(Et j'écris consolé : «Au commencement était l'action »)

Wittgenstein, De la Certitude.

\section{Introduction}

L'étude du langage, au cœur de la réflexion philosophique depuis Platon, s'est considérablement renouvelée à l'aube du $\mathrm{XX}^{\mathrm{e}}$ siècle par l'invention de la linguistique par Saussure et l'apparition des langages logiques, totalement formalisés et symboliques. Je voudrais aujourd'hui contribuer à nos échanges interdisciplinaires en insistant sur un autre aspect des recherches contemporaines en philosophie du langage : l'avènement progressif du paradigme actionnel qui appréhende les phénomènes discursifs et communicationnels en termes d'action et impose une approche résolument pragmatique.

Je commencerai par rappeler l'histoire ${ }^{1}$ de ce j'ai nommé naguère « le tournant actionnel » [Vernant, 1997]. Celui-ci, préfiguré par les intuitions de Frege, se concrétise avec Austin, Grice et Wittgenstein. Je montrerai ensuite que ce nouveau paradigme requiert l'élaboration d'une théorie générale de l'action dont j'esquisserai les principaux concepts. J'en viendrai enfin à une conception résolument actionnelle du dialogue comme interaction langagière soumise à des finalités transactionnelles à la fois intersubjectives et intramondaines.

\section{Le paradigme actionnel}

\section{$\underline{\text { A-Frege }}$}

On trouve les prémices de la nouvelle approche actionnelle du langage dans les dernières Recherches logiques de Frege [1918] $]^{2}$ où le logicien introduit le concept de

1. Il faut entendre ce terme au sens de récit [story]. Mon objectif n'est ici pas de faire l'historique précis de l'apparition de ce nouveau paradigme, mais d'en pointer les scansions philosophiques majeures. Ainsi ne suivrai-je pas la chronologie en adoptant la série : Frege, Austin, Grice puis Wittgenstein. Quant au point de départ, on peut parfaitement le discuter, cf. note 2. Ainsi ai-je montré ailleurs, en procédant à une analyse de la genèse du concept d'assertion, que Russell avait esquissé dès le tout début du précédent siècle une approche proprement pragmatique du concept de dénégation, cf. [Vernant, 2003b].

2. On pourrait tout aussi bien remonter à Peirce dont l'analyse pragmatique de l'assertion en termes d'acte engageant la responsabilité du locuteur à l'égard de la vérité préfigure les développements de la théorie 
force assertive pour expliquer la spécificité des jugements.

Ayant caractérisé la science comme usage méthodique de questions, Frege définit la compréhension d'une question comme la « saisie d'une pensée ». Par exemple, la question « Socrate est-il mort ? » commande la saisie de la pensée selon laquelle Socrate est mort ou bien selon laquelle Socrate n'est pas mort. Logiquement, si la pensée sous sa forme affirmative se symbolise par $« p »$, sa saisie se marque par le signe «-p », le trait horizontal indiquant la simple considération d'un contenu de sens, i.e. d'une pensée.

Une question n'est pas une réponse : "L'être d'une pensée ne consiste pas dans son être vrai [Wahrsein]. Et il faut admettre des pensées au sens proposé, puisque la science fait méthodiquement usage de questions »[Frege, 1918, 197]. La connaissance n'apparaît que secondairement avec la réponse énoncée par une phrase qui, ayant force assertive [behauptende Kraft], exprime un jugement. Le jugement, en nous faisant passer de la simple saisie de la pensée à sa valeur de vérité, engage seul la reconnaissance de la vérité : «Je tiens que le jugement est non pas la simple saisie d'une pensée, mais la reconnaissance de sa valeur de vérité » [Frege, 1892, 110, note 2]. Logiquement, ceci se traduit précisément par le signe d'assertion (littéralement signe de jugement : Urteilsstrich), tiret vertical ajouté à gauche du tiret horizontal de contenu. La réponse « Socrate est mort » se symbolise alors par « $\vdash p$ » : La réponse à une question est une assertion, fondée sur un jugement, que la question reçoive une réponse positive ou négative » [Frege, 1918, 195, trad. modifiée].

QUESTION : $\quad$ Socrate est-il mort?

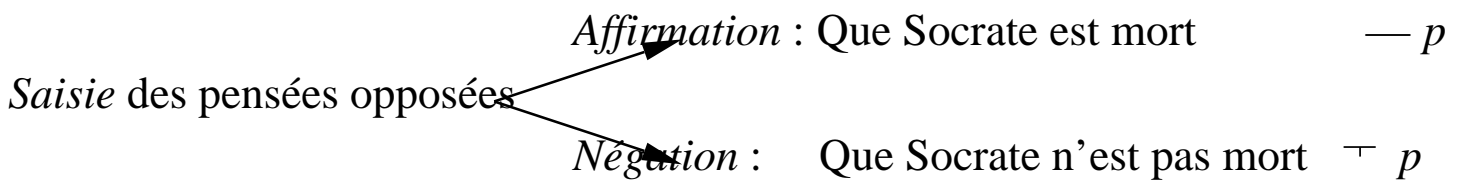

RÉPONSE : Assertion: $\quad$ Socrate est mort $\vdash p$

On ne soulignera jamais assez le caractère éminemment pragmatique de cette analyse proposée dès 1918 par le grand logicien. D'abord, la question du jugement logique est explicitement appréhendée dans ce que Michel Meyer [1986] appellerait un contexte problématologique de recherche de connaissance par question/réponse. La construction du savoir relève bien d'un processus dialogique. De plus et surtout, le jugement est bien défini comme un acte ayant une force spécifique. Frege d'ailleurs y insiste en précisant que : «On suivra parfaitement l'usage si l'on entend par jugement l'acte de juger, comme un saut est l'acte de sauter. .../... Si le jugement est un acte, il 
acteur, et l'acte n'est pas entièrement connu si l'acteur n'est pas connu » [Frege, 1918, 205, note 1]. La dimension actionnelle de l'analyse est ainsi clairement assumée même si elle n'est pas développée pour elle-même.

\section{$\underline{B-\text { Austin }}$}

Il reviendra au philosophe d'Oxford John Austin, traducteur anglais des Grundlagen der Arithmetik de Frege, de poursuivre cette analyse. Comme ses travaux sont bien connus, je voudrais simplement rappeler et délimiter son apport spécifique.

Austin porta un coup décisif au présupposé représentationnel en dénonçant l'illusion descriptive [descriptive fallacy] qui gouvernait jusqu'alors l'appréhension du langage. Prenant le relais du préjugé descriptif de l'époque classique, l'approche logique moderne avait continué à assigner au langage une fonction essentiellement cognitive de description du monde. La question demeurait celle de la vérité du discours conçue comme adéquation du dit au fait. À côté de cet usage purement constatif du langage - ex. «Le ciel est bleu », « $2+2=4 »$, etc. - Austin insista sur un usage performatif consistant à utiliser les mots pour produire une action. Les énonciations : «Je baptise ce navire le Queen Mary », «Je vous déclare mari et femme », «J'ouvre la séance », «Je déclare la guerre », etc., réalisent des actions que l'on fait par le langage : « dire une chose, c'est la faire » [Austin, 1962, 13].

Loin de considérer qu'il s'agissait là d'un usage second, voire parasitaire du langage, Austin inversa le rapport en soutenant que tout usage effectif du langage avait valeur d'authentique acte. Loin de s'avérer canonique, l'usage purement représentatif, descriptif, n'était plus qu'un type d'acte de discours parmi d'autres, soumis comme d'autres à des conditions de succès. Tout dire devenait un faire. Tirant systématiquement les conséquences de l'approche actionnelle du discours inaugurée par Frege, Austin distingue entre signification locutoire et force illocutoire, symbolisable par $F(p)$. Correspondant à la simple saisie de la pensée, la signification locutoire s'analyse en termes frégéens de sens et de référence, d'articulation entre procédures référentielles et prédicatives [Austin, 1962, 108]. Et l'idée de force illocutoire constitue une généralisation du concept frégéen de force assertive ${ }^{3}$ : à une même signification locutoire peuvent correspondre différentes forces. Ainsi en est-il de « La porte est fermée » qui a force assertive, de «Fermez la porte ! qui a force directive d'ordre, etc. Désormais, toute énonciation s'interprète en termes d'acte de discours [speech act] produit par un locuteur déterminé en un contexte spécifié. Les conditions de vérité régissant le contenu propositionnel, loin d'être abstraitement appréhendées, sont tributaires de conditions de succès [felicity conditions] qui gouvernent l'acte de

3. À cet égard, il est regrettable que le traducteur ait cru bon de traduire «force » par « valeur», 
discours en sa totalité. Austin s'attache à cerner les différents types d'échec des actes de discours ${ }^{4}$. Par exemple, l'assertion échoue si le locuteur ne croit pas ce qu'il dit : elle est insincère. On retrouve ici G.E. Moore qui remarquait ${ }^{5}$ qu'il est pragmatiquement paradoxal d'asserter : «La chatte est sur la natte, mais je ne le crois pas ». Affirmer « La chatte est sur la natte $»^{6}$ a pour condition la croyance $d u$ locuteur en ce qu'il énonce. Le sens de l'énoncé paradoxal contredit dans sa dernière partie ce qu'implique pragmatiquement la force d'assertion de sa première partie. S'ouvre ainsi la question du mensonge à propos de laquelle nous avons consacré des analyses que nous ne reprendrons pas ici [Vernant, 1997, ch. 4].

La dimension actionnelle des travaux d'Austin est patente. Un acte de discours est bien d'abord un acte susceptible de réussir ou d'échouer. D'ailleurs Austin admet des actes de discours non discursifs. La contradictio in adjecto n'est qu'apparente car il est manifestement possible de communiquer quelque chose sans passer par le discours en recourant à des mimiques, gestes, postures ou autres actions purement physiques. L'acte communicationnel ne se réduit pas à l'action verbale. Je peux désapprouver verbalement ou en lançant une tomate : «Si vous lancez une tomate dans une réunion politique (...), la conséquence sera probablement de faire connaître aux autres que vous désapprouvez, et de les amener à penser que vous avez certaines convictions politiques »[Austin, 1962, 120]7 . Ceci me conduit à préférer le terme de langagier entendu au sens le plus large à celui de discursif : il existe bien un langage des mimiques, des gestes, des regards, etc. qui déborde manifestement la seule expression discursive, verbale.

À cela s'ajoute le fait qu'Austin prend explicitement en compte les conséquences de l'action discursive sur l'auditeur à travers le niveau, proprement praxéologique, des objectifs (consciemment visés) et des suites perlocutoires (produites) 8 .

4 . Les conférences 2 et 3 proposent six conditions de succès mettant en jeu : $1^{\circ}-$ le respect de la procé-

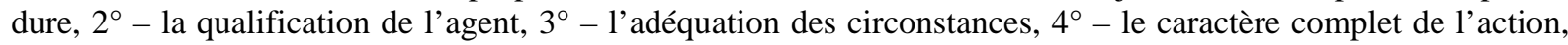
$5^{\circ}$ - la sincérité des intentions, $6^{\circ}-$ le comportement ultérieur.

5. Cf. [Moore, 1942, 542-3] et [Daval, 1997, chap. 7].

6 . Nous reprenons la traduction de «The cat is on the mat » proposée par [Armengaud, 1985, 73].

7 Austin précise : «[des actes illocutoires] peuvent être exécutés sans qu'on use de paroles ; mais même alors, l'acte (l'avertissement, par exemple) doit être un acte non verbal conventionnel pour mériter d'être appelé illocutoire » [Austin, 1962, 129]. [Austin, 1962, 135] oppose parler et employer un bâton : en ce dernier cas, la menace ne relève pas de la convention.

8 . Ce niveau perlocutoire ne relève pas de règles conventionnelles, mais de dispositifs contingents liés aux statuts des interlocuteurs, au problème, à la situation, etc. Plutôt de l'écarter indûment comme le fait [Searle, 


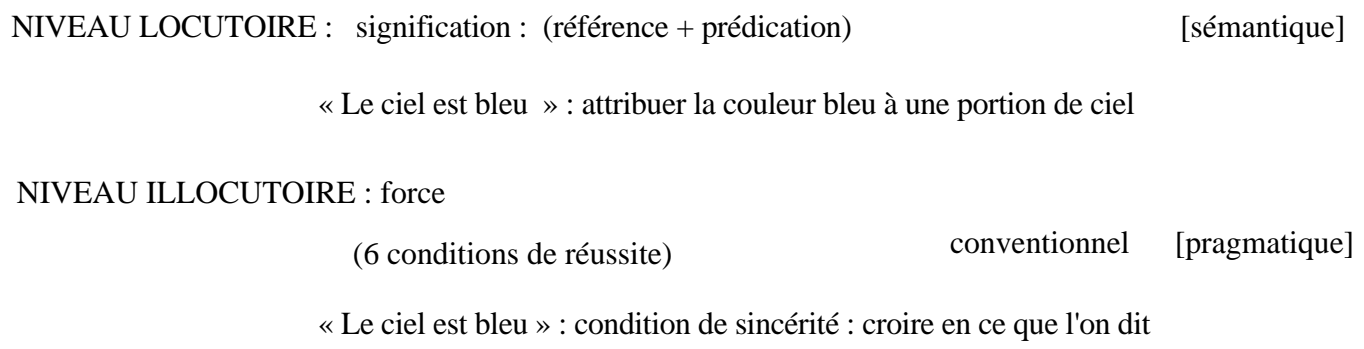

Remarquons enfin qu'Austin inscrit explicitement son analyse du langage ordinaire dans le cadre plus large d'une théorie générale de l'action : "Reste une objection à nos actes illocutoires et perlocutoires, selon laquelle la notion d'acte n'est pas claire. Nous y répondons par une théorie générale de l'action » [Austin, 1962, 117]. On notera qu'après cette déclaration, Austin tente bien de définir et de distinguer action discursive et action physique, mais qu'il est loin d'avoir donné une définition satisfaisante de l'action en général. Toute la question - qui demeure encore la nôtre - est de définir le concepts fondamentaux d'une théorie générale de l'action, d'une praxéologie qui fournisse le cadre conceptuel d'une analyse authentiquement pragmatique des phénomènes communicationnels.

\section{$\underline{C-\text { Grice }}$}

Malgré leur indéniable apport inaugural, les analyses du langage ordinaire proposées par Austin souffrent d'un défaut congénital (qui d'ailleurs perdure dans la théorisation de Searle et dont Daniel Vanderveken [1999] tente de se départir) : leur caractère étroitement monologique. L'acte de discours est acte du seul locuteur qui demeure maître et possesseur du sens. Bien entendu, l'acte de discours vise l'« auditeur ». Mais celui-ci est réduit au rôle passif de récepteur de l'acte, chargé de comprendre l'intention initiale du locuteur'. À cela s'ajoutent les objectifs perlocutoires de l'acte illocutoire, c'est-à-dire les effets attendus de l'acte sur autrui. Une menace peut, par exemple, viser à effrayer l'auditeur.

Incidemment, Austin note bien que certains actes de discours du locuteur, qu'il nomme actes contractuels, requièrent la complémentarité d'une réponse de l'auditeur.

9. Cf. [Austin, 1962, 124] « L'exécution d'un acte illocutoire inclut donc l'assurance d'avoir été bien 
C'est typiquement le cas de l'échange des consentements durant la cérémonie du mariage ou de l'accord qui vient sceller un pari ${ }^{10}$. Malheureusement, il ne s'agit là que d'exceptions qui ne mettent pas en question le caractère foncièrement monologique de l'analyse inaugurale des actes de discours.

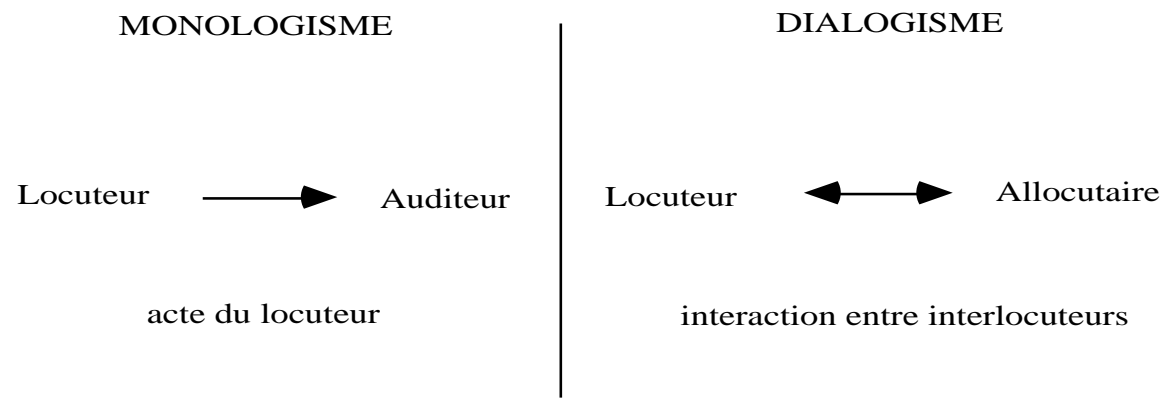

Il revient à Paul Grice d'avoir fourni les linéaments d'une approche véritablement interactionnelle de la communication. Dans son article fameux publié en 1975 «Logic and Conversation », il introduisit un nouvel objet d'analyse pragmatique : la « conversation » qu'il caractérisait de façon très large comme tout phénomène d'échange discursif. Il faisait l'hypothèse que la « conversation »- ou, au sens français du terme, le dialogue ${ }^{11}$ - constitue une des formes d'interaction rationnelle de l'homme. Elle se produit entre des agents humains tenus pour rationnels en ce qu'ils agissent en fonction de fins et selon des règles. Dès lors, qu'elle soit étroitement finalisée ou non, elle répond à un principe de coopération : " Nos échanges de paroles ne se réduisent pas en temps normal à une suite de remarques décousues, et ne seraient pas rationnels si tel était le cas. Il sont le résultat, jusqu'à un certain point au moins, d'efforts de coopération ; et chaque participant reconnaît dans ces échanges (toujours jusqu'à un certain point) un but commun ou un ensemble de buts, ou au moins une direction acceptée par tous. Ce but ou cette direction peuvent être fixés dès le départ (par ex. par la proposition initiale de soumettre une question à la discussion), ou bien peuvent apparaitre au cours de l'échange ; ils peuvent être relativement bien définis, ou assez vagues pour laisser une latitude considérable aux participants (comme c'est le cas dans les conversations ordinaires et fortuites). Mais à chaque stade certaines manœuvres conversationnelles possibles seraient en fait rejetées comme inappropriées du point de vue conversationnel. Nous pourrions ainsi formuler en première approximation un principe général qu'on s'attendra à voir respecté par tous les participants : que votre contribution conversationnelle corresponde à ce qui est exigé de vous, au

10. Cf. [Austin, 1962, 65]. «Ma tentative de faire un pari en disant "Je vous parie six pence" échoue, par exemple, à moins que nous ne disiez "d'accord" (ou des paroles à peu près équivalentes) ». La même chose vaut pour l'acte de donner un cadeau, nommer quelqu'un, etc. Est en cause l' « accroc » dans la classification des cas d'échec. Ainsi, l'interaction n'a pas valeur générale.

11. Au sens anglais, « conversation » vaut généralement pour toute forme d'entretien, de dialogue. 
stade atteint par celle-ci, par le but ou la direction acceptés de l'échange parlé dans lequel vous êtes engagé » [Grice, 1975, 61]. De ce principe découlent quatre règles, ou maximes qui déterminent les modalités de la coopération :

$1^{\circ}$ - de quantité : que votre contribution ne contienne ni trop ni trop peu d'information,

$2^{\circ}$ - de qualité : que votre contribution soit véridique (ceci rappelle la condition de sincérité de la théorie des actes de discours),

$3^{\circ}-$ de relation : que votre contribution soit pertinente [relevant],

$4^{\circ}$ - de modalité : que votre contribution soit claire (évitez obscurité et ambiguité). Naturellement, ces règles peuvent être respectées ou non. On distinguera trois cas de non-respect :

A - l'interlocuteur refuse « de jouer le jeu », la conversation ne peut se poursuivre.

$\mathrm{B}$ - l'interlocuteur a du mal à concilier toutes les règles, il est amené involontairement à en violer une. On peut être conduit à donner trop peu d'informations (viol de la règle 1) pour ne pas en dire plus que ce que l'on sait (respect de la règle 2). C'est par exemple le cas d'un scientifique qui lors d'un entretien télévisé refuse de s'engager au-delà de son champ de compétence.

$\mathrm{C}$ - l'interlocuteur transgresse intentionnellement et ostensiblement l'une des règles. Ce dernier cas de transgression volontaire est intéressant parce qu'il introduit une stratégie indirecte de communication. Ce que veut dire le locuteur n'est pas réductible à ce qu'il dit explicitement. Le sens de l'intervention n'est plus fournie directement et manifestement par la signification, mais indirectement par le truchement d'une inférence qui s'appuie sur l'acceptation du principe de coopération et de ses règles : l'implicitation conversationnelle.

Il s'agit de faire comprendre ce que l'on veut dire sans avoir à le dire. L'écart est ici manifeste entre la signification littérale (sémantique) et le sens communiqué (pragmatique) qui est inféré par une procédure spécifique (non logique au sens d'implication) : «Un individu qui, parce que (en même temps que) il déclarait (ou faisait semblant de déclarer la proposition $P$, a implicité $Q$, peut être considéré comme ayant émis délibérément dans la conversation l'implicitation $Q$ aux conditions suivantes : $1^{\circ}$. Il faut d'abord qu'il n'y ait pas lieu de supposer qu'il n'observe pas les règles de la conversation, ou au moins le principe de coopération. $2^{\circ}$. Il faut ensuite supposer que l'individu en question sait ou pense que $Q$ est nécessaire pour que le fait qu'il dise (ou fasse semblant de dire) $P$ (ou le dise précisément en ces termes) ne soit pas contradictoire avec la supposition 1. $3^{\circ}$. Le locuteur pense (et s'attend à ce que l'interlocuteur pense que lui pense) que l'interlocuteur est capable de déduire ou de saisir intuitivement qu'il est absolument nécessaire de faire la supposition évoquée en $2 »[$ Grice, 1975, 64]. 
Prenons un exemple. Si $A$ rédige une recommandation pour l'un de ses étudiants qui postule en philosophie en écrivant : «M. $X$ a une maîtrise remarquable du français, et il a été assidu à mes cours. Je vous prie d'agréer, etc. ». A ne refuse pas de jouer le jeu, puisque, s'il ne voulait pas coopérer, il pourrait fort bien ne pas écrire du tout, mais il n'ignore pas qu'on lui demande plus d'informations. Le destinataire en inférera que $A$ implicite l'information selon laquelle M. $X$ n'a pas les qualités philosophiques requises pour le poste convoité. Ainsi, le sens effectivement communiqué ne correspond pas à la signification exprimée et requiert une inférence non plus logique (implication), mais pragmatique (implicitation) qui s'appuie sur la connaissance des règles de la conversation (Principe de coopération), sur le contexte extra-linguistique et sur les intentions supposées de l'interlocuteur.

\section{IMPLICITATIONS CONVERSATIONNELLES}

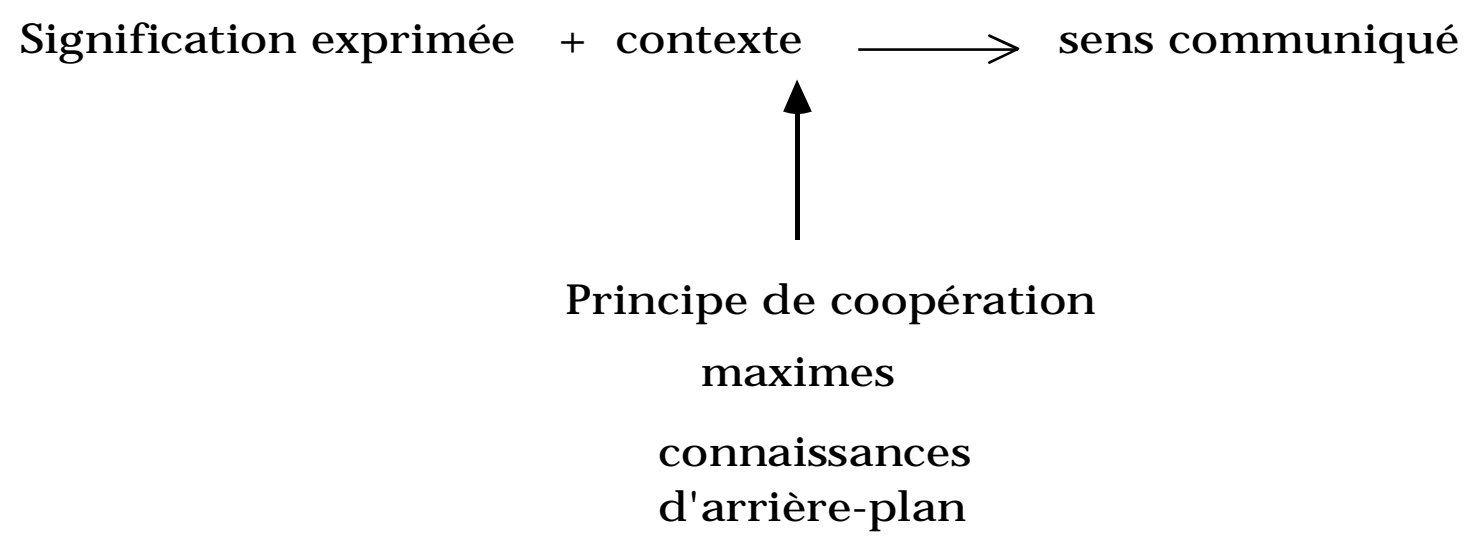

Cette théorie des implicitations conversationnelles inaugure une approche authentiquement pragmatique de la dimension proprement inférentielle du sens. Dans le contexte naturel de la communication, le sens déborde largement la signification littérale de la phrase prononcée pour relever d'inférences pragmatiques. Sperber et Wilson [1989], dans la même perspective réduiront toutes les maximes à la seule pertinence et montreront que cette pertinence régit toutes les inférences communicationnelles.

Mais pour ce qui nous concerne ici, c'est une fois de plus la dimension proprement actionnelle clairement assumée qui nous importe. Grice note explicitement que son analyse des processus d'interaction verbale vaut aussi bien pour les phénomènes de transactions non verbales : "Si quelqu'un passe quand je me débats avec ma voiture en panne, il ne fait aucun doute que je m'attends quelque peu à ce qu'il m'offre son aide ; mais une fois qu'il s'est mis avec moi à bricoler sous le capot, les attentes se renforcent et se précisent (à condition que rien ne me fasse a priori douter de ses compétences) ; et les échanges parlés me semblent offrir certains traits caractéristiaues communs à l'ensemble des transactions coopératives : 
$1^{\circ}$ ) Les participants ont un but commun immédiat, par exemple réparer une voiture ; leurs buts ultimes peuvent bien sûr être indépendants et même conflictuels - chacun peut vouloir réparer la voiture pour s'en aller avec et laisser l'autre sur le bord de la route. Dans les échanges parlés typiques, il y a un but commun même si, lorsque deux voisins bavardent, ce but commun est de second ordre : chacune des parties en présence doit momentanément s'identifier avec les intérêts passagers de l'autre.

$2^{\circ}$ ) Les contributions des participants doivent s'imbriquer et dépendre l'une de l'autre.

$3^{\circ}$ ) Il est plus ou moins sous-entendu (explicitement parfois, mais souvent tacitement) que, toutes choses égales par ailleurs, la transaction doit continuer dans le style qui lui convient, à moins que les deux parties ne tombent d'accord pour y mettre fin. On ne peut pas prendre le large ou se mettre à faire autre chose comme ça » [Grice, 1975, 63]. On a bien là l'esquisse d'une définition de l'action conjointe, catégorie praxéologique à laquelle appartiennent aussi bien les interactions communicationnelles que les transactions non communicationnelles.

À cela s'ajoute le fait que Grice fournit une traduction transactionnelle de ses maximes de conversation :

«Quantité : Si quelqu'un m'aide à réparer une voiture, je m'attends à ce que sa contribution ne corresponde ni plus ni moins qu'à ce qui est demandé ...

Qualité : J'attends une aide véritable, pas un semblant d'aide ...

Relation : Je compte sur une aide de mon associé ajustée aux besoins immédiats de chaque stade de la transaction ...

Modalité : Je compte que mon partenaire élucide pour moi la nature de sa contribution et qu'il l'accomplisse en un temps raisonnable » [Grice, 1975, 62].

\section{$\underline{D-\text { Wittgenstein }}$}

Après Frege, Austin et Grice, il importe de mentionner Wittgenstein parmi les grands précurseurs de l'approche actionnelle du langage. Vous aurez compris qu'il s'agit bien entendu de ce qu'il est convenu d'appeler le « second » Wittgenstein, l'auteur des Investigations philosophiques et de son dernier ouvrage De la Certitude.

Dès les années 1935, récusant l'approche étroitement logiciste du Tractatus, Wittgenstein introduit le concept de jeu de langage dont le premier exemple donné au paragraphe 23 des Investigations philosophiques est : "Commander et agir d'après un commandement ». Par anticipation, Wittgenstein dépassait le monologisme d'Austin : on ne peut concevoir un ordre sans l'acte d'obéissance qui le satisfait, une question sans sa réponse, etc. On peut alors interpréter les analyses de Grice comme une première conceptualisation de cette notion protéiforme de jeu de langage que Wittgenstein se refuse à définir. La question est alors de savoir pourquoi revenir à Wittgenstein après Grice. Tout simplement parce que Wittgenstein introduit explicitement la ques- 
tion des relations entre ce que je nomme interaction et transaction, c'est-à-dire entre les jeux de langage et les formes de vie qui leur donnent sens : " Le mot "Jeu de langage" doit faire ressortir ici que le parler du langage fait partie d'une activité ou d'une forme de vie » [Wittgenstein, 1969, § 23]. Là encore Wittgenstein n'est pas très précis sur le sens à donner à cette notion ultime de forme de vie, mais il insiste bien sur la subordination des jeux de langage à ces formes de vie. Évitant toute réduction logocentrique de l'analyse, il souligne ce que je nomme l'hétéronomie des interactions langagières [Vernant, 1997, 97]. Les jeux de langage ne peuvent fonctionner par euxmêmes, en quelque sorte dans le vide. C'est bien l'action qui assigne finalité au langage : « Ce que nous disons reçoit son sens du reste de nos actions » [Wittgenstein, $1969, \S 229$ ], et ce sont les modalités collectives de transaction qui assignent finalité aux interactions communicationnelles : « Le terme, c'est notre action qui se trouve à la base du jeu de langage » [Wittgenstein, 1969, § 229]. En fait, Wittgenstein substitue à l'antique question du sens non pas celle de l'usage linguistique, mais bien celle de l'utilisation (Gebrauch) du langage à des fins actionnelles. En ce sens, les intuitions du « second » Wittgenstein demeurent fécondes. La question est de les conceptualiser et de les théoriser.

On le constate, le bouleversement opéré de Frege à Wittgenstein en passant par Austin et Grice est profond. À l'épistémè de la représentation qui, à l'âge classique, se fondait sur un primat de la pensée et du sujet pensant, reléguant l'expression langagière à un rôle second et subalterne, a succédé au début du $\mathrm{XX}^{\mathrm{e}}$ siècle, avec notamment l'apparition de la linguistique et de la logique symbolique, une épistémologie du signe et des créations symboliques de l'Homme. Mais se préparait déjà dans le champ du langage comme dans d'autres - que l'on pense en psychologie à Vygotski et Piaget -, une ultime modification consistant assurer un primat de l'action sur le signe. Il nous reste alors, comme le notait d'ailleurs Wittgenstein à prendre l'adage faustien «Au commencement était l'action » au sérieux. Pour notre part, nous considérons qu'il importe d'abord de réactiver les études praxéologiques inaugurées par le Français Alfred Espinas dès 189712 .

Dans le temps imparti, mon objectif ne peut pas être de développer cette approche actionnelle afin d'en établir la puissance analytique. Je me contenterai d'insister sur ce bouleversement sourd mais crucial qui, à l'aurore du nouveau millénaire, augure peut-être d'une nouvelle épistèmé.

12. Alfred Espinas (1844-1922) fut professeur à la faculté des Lettres (1880) puis Doyen de l'université de Bordeaux (1887) où il obtint la création d'une chaire de " pédagogie et science sociale » dont le premier titulaire fut Émile Durkheim. En 1894, on le préféra à Durkheim pour la chaire d'histoire économique et sociale à la 


\section{De l'action}

\section{$\underline{\text { A-La nécessité d'une praxéologie }}$}

Qu'elle soit comprise comme acte de discours ou comme jeu de langage, l'action communicationnelle, tout en possédant sa spécificité, constitue une forme d'action. Comme l'avait indiqué Austin, le paradigme actionnel qui régit la pragmatique de la communication requiert une théorie générale de l'action, une praxéologie. Disons le tout de suite une telle théorie de l'action est fort loin d'être achevée. Le concept d'action, pourtant introduit dès Aristote, est sans doute l'un des plus délicats et nous ne disposons pas actuellement d'une définition satisfaisant et opératoire d'un tel concept. Je ne peux ici aborder directement cette question de la construction d'une praxéologie. J'ai tenté d'y contribuer en proposant une définition minimale de l'action, de l'agent, ainsi que des types d'actions collectives.

\section{Définition minimale de l'action}

Est d'abord requise une définition opératoire de l'action. Ce point est sans doute le plus délicat dans la mesure où la philosophie de l'action est en pleine effervescence. L'action, comme mode d'intervention sur le monde, possède nécessairement une dimension physique, corporelle. La question est alors de faire le départ entre un phénomène physique tenu pour un simple événement et un autre (ou éventuellement le même) considéré comme une authentique action. Or aucune caractéristique propre au phénomène physique ne permet de trancher cette question.

À titre d'hypothèse, j'admets pour critère discriminant entre événement et action l'attribution de l'origine du phénomène physique à un agent doué d' « intelligence » et, partant, capable de conduire et contrôler l'action ${ }^{13}$. Pour peu que l'on maintienne une neutralité des termes en tentant d'éviter tout anthropomorphisme, une telle approche permet une définition minimaliste de l'action. Ainsi, l'intelligence ne signifie pas nécessairement capacité de représentation, de réflexion et de conceptualisation, mais peut s'entendre plus élémentairement comme l'aptitude à un simple échange informationnel avec le milieu dans lequel se produit l'action. La conduite peut s'interpréter comme la capacité d'engager et de maintenir l'action lorsque celle-ci se déploie dans le temps. La décision d'agir et la direction de l'action se font selon un but, une finalité qui ne sont pas nécessairement conscients ni prédéterminés. Est en cause essentiellement le caractère téléologique de toute action. Enfin, le contrôle de l'action fait intervenir la capacité de rectifier, suspendre ou interrompre éventuellement le cours de l'action. Un tel contrôle peut passer par une décision volontaire et délibérée ou par un

\footnotetext{
13. Notre position est ici proche de celle de Harry G. Frankfurt : « Le problème de l'action » in [Neuberg
} 
simple processus d'homéostasie. Dès lors, peuvent être appréhendés des types fort différents d'agents : les systèmes artificiels tels les robots, e.g. les «créatures » de Rodney Brooks ${ }^{14}$, les organismes animaux aussi bien que les Hommes ${ }^{15}$. Mais comme notre objet est l'examen des rapports entre le discours, phénomène spécifiquement humain, et l'action, nous poursuivrons notre définition de l'action en caractérisant un type particulier d'agent : la personne humaine.

\section{La personne comme agent}

Il convient de caractériser la personne comme un agent particulier. Sans pouvoir ici reprendre le détail de l'analyse [Vernant, 1997, ch. 8], précisons simplement que je définis l'agent comme un être possédant quatre caractéristiques :

$1^{\circ}$ la réflexivité : sa capacité d'avoir conscience de soi, des autres et des mondes.

$2^{\circ}$ la rationalité : sa capacité d'agir à partir d'une planification réglée,

$3^{\circ}$ la finalité : sa capacité d'agir selon des fins et des valeurs,

$4^{\circ}$ la coopérativité : sa capacité de participer à une action collective supposant la mise en œuvre d'une stratégie commune.

\section{Les types d'action}

Enfin est nécessaire une typologie des actions qui permette notamment de distinguer les formes de l'action collective. Je propose de distinguer entre action singulière, isolée et actions collectives, ces dernières pouvant être des actions plurielles, simplement parallèles, ou communes, la même action est alors faite pas plusieurs ou proprement conjointes supposant l'acceptation d'un même but et la coordination d'actions différentes et complémentaires.

14. Rappelons que ces « créatures » n'ont pas l'intelligence représentationnelle des mondes dans lesquels elles évoluent, cf. [Vernant, 1997, ch. 7].

15. La question se pose du statut des « agents » informatiques en Intelligence artificielle distribuée. La terminologie et la thématique en la matière ne sont pas encore complètement fixées. Dans la mesure où les enjeux sont essentiellement informationnels, on pourrait les considérer comme des agents exclusivement capables d'interactions communicationnelles. Ainsi, [Ferber, 1995, 14-17] les appelle « agents purement communicants », ou « agents logiciels ». Mais on retombe alors sur la question des capacités sémantiques des machines, cf. 


\begin{tabular}{|c|c|c|c|c|}
\hline $\begin{array}{l}\text { TYPE } \\
\text { D'ACTION }\end{array}$ & BUT & STRATEGIE (S) & $\operatorname{ACTE}(\mathbf{S})$ & EXEMPLE \\
\hline Singulière & individuel & personnelle & isolé & $\begin{array}{l}\text { Jouer du } \\
\text { piano seul }\end{array}$ \\
\hline Plurielle & $\begin{array}{l}\text { même but } \\
\text { imposé }\end{array}$ & personnelle & différents & $\begin{array}{l}\text { Sauve } \\
\text { qui peut }\end{array}$ \\
\hline \multicolumn{5}{|l|}{ Collective : } \\
\hline Commune & $\begin{array}{l}\text { même but } \\
\text { accepté }\end{array}$ & identique & identiques & $\begin{array}{l}\text { pousser } \\
\text { une voiture }\end{array}$ \\
\hline Conjointe & $\begin{array}{l}\text { même but } \\
\text { accepté }\end{array}$ & coordonnées & différents & $\begin{array}{l}\text { Jouer aux } \\
\text { échecs }\end{array}$ \\
\hline
\end{tabular}

\section{$\underline{B-\text { Action/Activité }}$}

Notre définition de l'agent comme réflexif, rationnel, finalisé et coopératif semble faire de l'action le résultat d'un choix délibéré, conscient et volontaire ; la réalisation d'une stratégie rationnellement réfléchie. Ceci témoigne de la façon dont on peut penser l'action humaine dans sa forme la plus sophistiquée et spécifique. Pour autant, il ne faudrait pas en conclure hâtivement que toute action humaine doive prendre cette forme, ni même qu'on décrit ainsi la forme effective du processus actionnel dans toute sa complexité.

On peut en effet modéliser les actions des Hommes, des animaux et même des robots en termes de représentations symboliques, de planification, de rationalité limitée. Ainsi Herbert Simon propose-t-il une approche exclusivement «symbolique » des actions humaines, cf. [Vera \& Simon, 1993]. Mais on peut lui reprocher de confondre le modèle avec le phénomène modélisé. Les partisans de l'« action située » proposent à l'inverse de concevoir l'activité effective comme un procès infra-symbolique de transaction entre l'agent et son environnement. William Clancey la définit ainsi : «L'activité située (situated activity) n'est pas une sorte d'action, mais de tout temps la nature de l'interaction animale, par opposition à la plupart des machines que nous connaissons. Il ne s'agit pas simplement de considérer que le contexte est important, mais que ce qui constitue le contexte, la manière dont vous catégorisez le monde, surgit dans sa relation (arises together) aux processus qui constituent l'activité de coordination physique. Être en train de percevoir le monde est agir en lui - pas dans une 
que ce que je suis en train de percevoir et que la manière dont je me déplace se codéterminent l'un l'autre » [Clancey, 1993, 95]16.

L'action peut être décrite et précisée par des règlements, des codes, des plans. Mais ce ne sont qu'abstractions par rapport à l'activité effective qui compose le procès transactionnel dans sa réalisation plénière. Il y a loin de la coupe aux lèvres et le plan calmement pensé et arrêté ne correspond que rarement à l'action effectuée in situ qui suppose à chaque instant ajustements et rectifications pour s'adapter opportunément aux aléas et imprévus des réactions du milieu et des autres agents. Là comme ailleurs, il convient, sans négliger ses mérites, de limiter les prérogatives de l'approche représentationnelle. De fait, l'homme peut rationaliser ses actions en recourant aux ressources symboliques, à la «grammaire » des jeux de langage. De façon prospective, il peut élaborer des plans et des stratégies pour construire rationnellement un projet. Inversement, il peut rétrospectivement donner un sens ou même justifier telle action en en fournissant une description rationnelle acceptable. Le psychanalyste pourra éventuellement déceler qu'il s'agit en fait d'une « rationalisation seconde »! Il demeure toutefois que l'activité, dans le temps même de son effectuation, constitue un processus ouvert, imprévisible et créatif qui dépend, en temps réel, d'une série de choix ponctuels et précis imposés par la conduite et le contrôle de la transaction en train de se faire. Si l'action peut être programmée, seule l'activité est réalisée. Si l'action peut se représenter, se planifier, se rationaliser et se justifier, l'activité ne peut que se réaliser, se produire : «Si dès lors j'ai épuisé toutes les justifications, me voici donc avoir atteint le roc dur et ma bêche se recourbe. Alors j'incline à dire "C'est ainsi que j'agis" » [Wittgenstein, 1953, § 217]. Une fois de plus, le terme échappe à toute discursivité pour relever d'attitudes, de propensions et de tropismes fondamentaux corporellement intégrés (Insistant sur le niveau biologique et perceptuel, [Varela, 1993] parle d'action « incarnée »). Il réside dans les schémas de transactions qui sont socialement et culturellement admis comme formes de vie.

Ayant fixé le cadre actionnel de l'analyse, nous pouvons désormais aborder la manière dont nous concevons les phénomènes communicationnels. Ce qui nous conduira d'abord à définir les interactions langagières pour ensuite les subordonner aux transactions extra-langagières qui leur donnent sens et finalité.

16. [Suchman, 1987, 50] introduit le terme situated action en référence principalement aux études ethnométhodologiques. Curieusement, elle cite Mead (p. 51), la théorie des indexicaux de Peirce (p. 58-62), puis la théorie des actes de discours (p. 39-42), mais à aucun moment elle n'explicite l'intérêt d'une approche pragma- 


\section{3 - L'interaction langagière}

Cette analyse praxéologique fournit le cadre général indispensable à une véritable approche actionnelle des phénomènes communicationnels. Il reste toutefois à montrer en quoi l'utilisation du discours constitue une action et en quoi cette action est spécifique en tant que communicationnelle.

Après avoir corrigé et complété dans la perspective actionnelle la typologie des actes de discours de Searle, nous avons tenté de préciser la définition, généralement floue, du phénomène dialogique, cf. [Vernant, 1997, ch. 3 \& 5]. Nous l'avons défini comme une activité conjointe et un processus interactionnel ouvert. Ceci suppose, techniquement la définition des interactes et des modèles projectifs de dialogue. J'appelle interaction langagière toute forme de communication dialogique qu'elle soit verbale ou non (par mimique, geste, regard, etc.). Cette interaction peut se définir à deux niveaux : celui élémentaire de l'interacte et celui englobant du modèle projectif du dialogue.

\section{$\underline{A-L ' \text { interacte }}$}

Ce que nous appelons interacte procède de la redéfinition dialogique de l'acte de discours. L'analyse sémantique des actes de discours relève d'une construction spéculative, philosophique et non d'une théorie linguistique qui prétendrait fournir la possibilité technique de déterminer la forme de surface des actes de discours. Il est manifeste que les modes grammaticaux - indicatif, interrogatif, impératif, optatif - ne fournissent pas de critères suffisants : un ordre n'est pas nécessairement à l'impératif, l'indicatif n'est pas réservé à l'assertion, etc. ${ }^{17}$. On peut envisager de recourir à d'autres critères, para-linguistiques, notamment prosodiques ${ }^{18}$. Mais, en vérité, il est proprement vain de prétendre assigner une quelconque forme canonique à chaque type d'acte de discours. Ce serait reproduire le monologisme natif de la théorie austinienne. Les actes de discours n'ont pas de réalité en soi, indépendamment du procès dialogique dans lequel ils acquièrent sens. La force illocutoire d'un acte de discours réside dans le fait qu'il prend valeur de coup dans un jeu de langage : ordonner n'a de valeur que par rapport à l'acte réciproque d'obéir. C'est cette intuition profonde du « second » Wittgenstein qu'il convient de conceptualiser ${ }^{19}$.

Dès lors, appréhendé dialogiquement, l'acte de discours s'avère proposition du lo-

17. « Nous pouvons poser une question à l'impératif ou à l'indicatif ("Dites-moi qui a gagné la course”, "J'aimerais bien connaître votre numéro de téléphone") ou émettre un ordre avec un indicatif ("Dans cette maison, nous enlevons nos chaussures avant d'entrer") », [Davidson,1984, 169].

18. [Colineau, 1997] montre bien les limites d'un tel critère. Fort justement, elle propose de déterminer de façon probabiliste la force illocutoire d'une énonciation à partir d'un ensemble de marqueurs, mais aussi d'informations contextuelles.

19. [Wittgenstein, 1953, § 2] considère un « langage absolument primitif » où un locuteur (le maçon) crie 
cuteur faite à l'allocutaire. Anticipant la réaction de l'allocutaire, le locuteur sélectionne $a b$ initio dans le champ des formes possibles une forme particulière qui, ne déterminant pas mécaniquement un seul type d'acte, laisse généralement une latitude d'interprétation à l'allocutaire. Par un mouvement de pro- et de rétroaction, les interlocuteurs parviennent à s'accorder dialogiquement sur une interprétation commune des actes émis [Trognon \& Brassac, 1992]. À partir de sa connaissance de l'allocutaire, de la situation commune, etc., le locuteur propose un acte et anticipe l'interprétation de l'allocutaire. On parle toujours pour, mais surtout par autrui. La réaction de l'allocutaire vient ou non confirmer cette anticipation. Si elle la confirme ou si l'écart est jugé par le locuteur minime ou sans conséquence pour la poursuite du dialogue, ce locuteur entérine implicitement l'interprétation de l'allocutaire. Si la rétroaction ne satisfait pas la proaction, le locuteur peut soit se rallier à l'interprétation de l'allocutaire, soit proposer une formulation nouvelle qui vise à rectifier l'interprétation initiale de l'allocutaire. C'est ce jeu d'aller-retour entre pro- et rétroaction qui définit dialogiquement l'acte de discours comme proprement une interaction produite conjointement par les deux interlocuteurs qui s'avèrent les colocuteurs de l'interacte.

NEGOCIATION DES ACTES DE DISCOURS

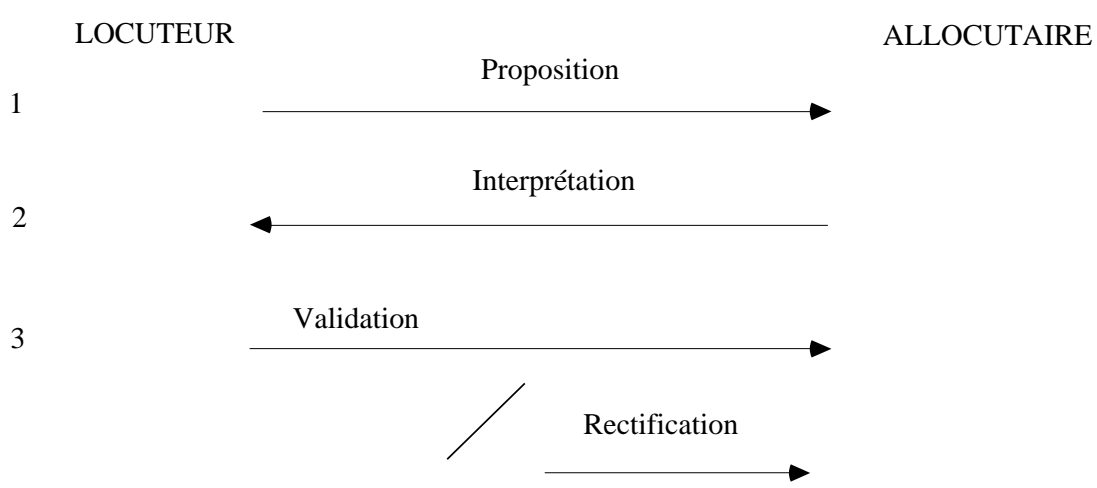

On comprend alors que l'analyse effective des forces illocutoires mises en jeu ne peut se faire qu' in situ, dans le cotexte d'un dialogue particulier, en prenant en compte une série complexe d'indices linguistiques, para-linguistiques, mais aussi en considérant le contexte interactionnel (type d'interaction, rôle dialogique des interlocuteurs, moment de l'interlocution, etc.). Sans pouvoir ici entrer dans les détails, considérons l'énonciation banale : «Pouvez-vous me donner l'heure ?». À ne prendre en compte in abstracto que sa seule forme, on exclura tout au plus qu'elle exprime un expressif, 
un promissif ou une déclaration ${ }^{20}$. Mais il n'est pas difficile d'imaginer divers contextes interactionnels dans lesquels cette énonciation peut acquérir différentes forces illocutoires. Imaginons la rencontre de deux passants dans la rue, notre énonciation peut susciter des réactions différentes qui lui assigneront à chaque fois une interprétation différente. On peut au moins envisager cinq cas.

- Cas 1 :

Passant $A$ : - Pouvez-vous me donner l'heure?

Passant $B$ :-Désolé, je n'ai plus de montre.

L'énonciation initiale est ratifiée par l'allocutaire comme une question directe sur ses possibilités effectives de fournir l'heure à laquelle il répond par la négative. Mais, on aurait pu tout aussi bien avoir :

- Cas 2 :

Passant $A$ :- Pouvez-vous me donner l'heure?

Passant $B:-$ Il est 18 heures.

Cette fois, l'indirection est prise en compte par l'allocutaire qui fournit immédiatement une réponse à la requête indirecte du locuteur. L'énonciation est alors entérinée comme la forme polie d'un ordre. Dans ce cas, la condition exprimée par la question directe est logiquement supposée remplie. On ne fournit normalement l'heure que si l'on dispose des moyens de la connaître. On peut envisager un cas où ceci est explicité.

- Cas 3 :

Passant $A:-$ Pouvez-vous me donner l'heure?

Passant $B:-$ Bien sûr, il est 18 heures.

Outre sa fonction phatique, le «Bien sûr » est une réponse explicite à la question directe. Quant à «Il est 18 heures », c'est la réponse à la requête indirecte.

On peut encore imaginer un cas plus complexe faisant appel à une montée métadiscursive.

- Cas 4 :

Passant $A:-$ Pouvez-vous me donner l'heure?

Passant $B:-$ Je ne vous la donne pas, je vous la prête : il est 18 heures. Feignant d'assimiler le temps à un objet que l'on pourrait posséder, l'allocutaire corrige le mot « donner» qui figurait dans la question initiale. Ce faisant, il inaugure une activité métadiscursive. Ensuite, en acceptant de «prêter » l'heure, il entérine implicitement la question directe : «Pouvez-vous ... ? ». Enfin, par sa réponse, il satisfait la requête indirecte. Dès lors, la réplique humoristique de cet allocutaire associe

20 Sur notre classification des actes de discours qui s'écarte notablement de celle de Searle, cf. [Vernant, 
les trois dimensions abstraitement ouvertes par l'énonciation initiale : la mention métalinguistique, la question directe, la requête indirecte.

Un dernier cas est toujours possible : celui du refus de répondre dans la mesure où une question est un directif moins contraignant qu'un commandement en ce qu'il offre toujours une possibilité de refus.

- Cas 5 :

Passant $A$ : - Pouvez-vous me donner l'heure?

Passante $B:-$ Excusez-moi, je n'ai pas le temps.

On peut supposer que l'allocutaire est ici une jolie jeune femme, pressée et lasse d'être importunée dans la rue. Elle interprète alors la question du passant non comme une réelle demande, mais comme un moyen fort peu original de l'aborder. Elle se dérobe en prétextant qu'elle est pressée. L'acte est alors disqualifié dans sa fonction fondamentale d'amorce d'une interaction. On peut aussi avoir la réponse impertinente : «Il est temps de vous acheter une montre »!

On résumera comme suit les cinq réponses possibles ${ }^{21}$, partant, les interprétations potentielles de l'acte initial :

21. On peut d'ailleurs envisager deux cas supplémentaires où le locuteur répond « oui » à la question directe sans fournir ensuite l'information souhaitée. Deux interprétations sont alors possibles. L'une humoristique où l'on feint de se satisfaire de la réponse positive à la question directe en négligeant, contre toute attente pragmatique, la réponse à la requête indirecte. C'est ce qui se produit dans le fameux sketch de Pierre Dac et Francis Blanche qui met en scène un pseudo-mage :

- Pouvez-vous le dire?

- oui,

- Vous pouvez le dire?

- oui!

- Bravo !, Il peut le dire !

Dans un contexte opposé, le fait de s'arrêter volontairement à la réponse « oui » à la question directe a valeur d'affront grave qui peut-être interprété comme : « Je possède l'information souhaitée, mais je ne vous la donne- 


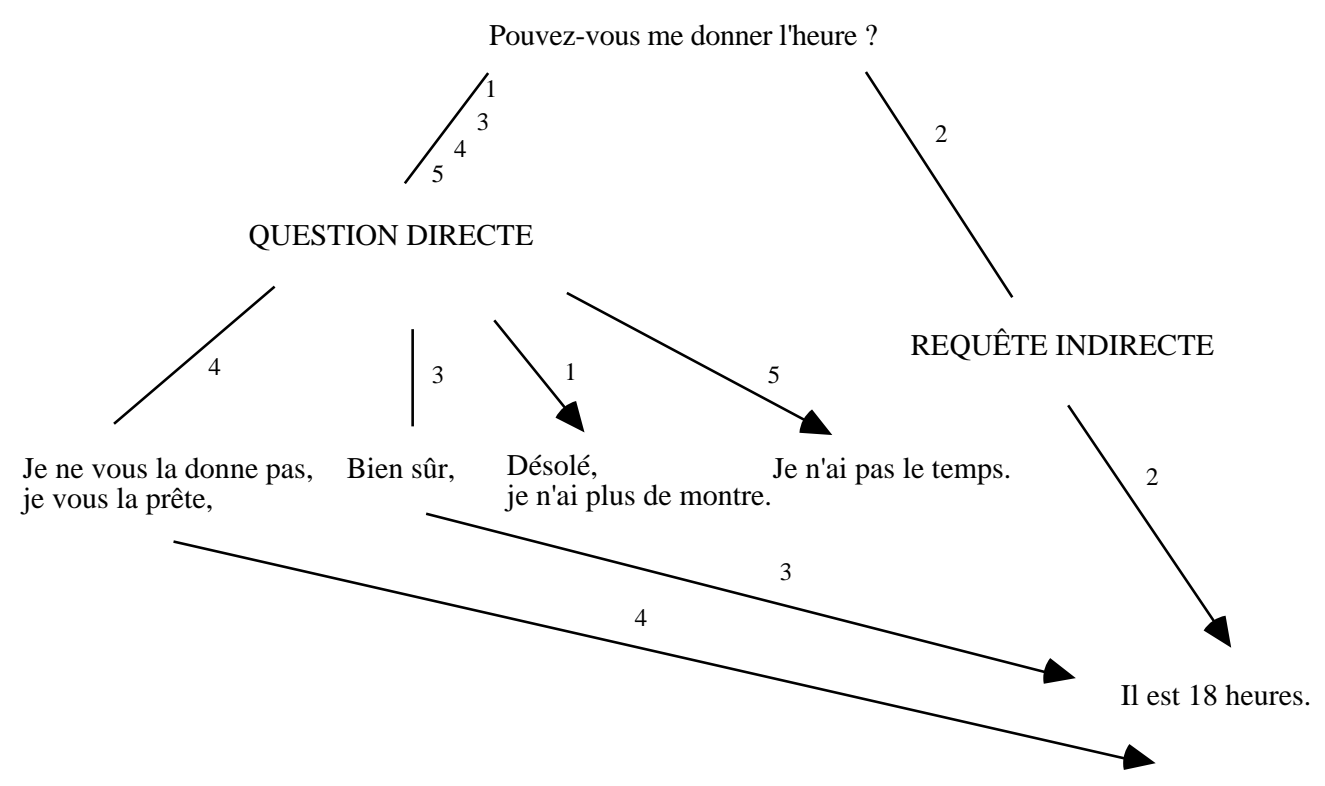

PLURIVALENCE INTERPRETATIVE D'UN INTERACTE

Ceci témoigne de la foncière plurivalence de la forme de surface des actes de discours. Cette plurivalence d'une énonciation donnée illustre clairement le fait que la force d'un acte de discours n'est déterminable que dans son interaction avec le (les) acte(s) qui le précède(nt) et/ou qui le(s) sui(ven)t. Un acte de discours constitue un coup dans un jeu qui suppose un avant-coup sous la forme des anticipations du locuteur à propos de l'allocutaire, et un après-coup au cours duquel l'allocutaire vient ou non ratifier les anticipations du locuteur. Sous peine d'incommunicabilité totale, un tel jeu est limité par les contraintes syntaxiques et sémantiques véhiculées par l'énonciation, mais il conserve toutefois une amplitude suffisante pour faire du dialogue une création potentiellement riche et sans cesse ouverte, une activité imprévisible. Ainsi, aucun acte de discours ne saurait constituer une action singulière, isolée et autonome. C'est toujours un élément d'une activité collective, précisément conjointe. Tout acte de discours doit être pensé comme interacte, et tout interacte comme l'un des échanges composant un dialogue, une interaction complexe.

\section{$\underline{B \text { - La modélisation projective de l'interaction }}$}

Sans pouvoir développer ici ce point, rappelons que nous envisageons tout dialogue comme une activité située, conjointe et un processus ouvert [Vernant, 1997, ch. 5 \& 8]. Dans sa réalité effective, tout dialogue qui s'engage est une aventure dont le devenir et l'achèvement dépendent de la situation, du lieu, du temps, des agents, de leurs finalités, du thème abordé, des stratégies successivement suivies, etc. Tout dialogue constitue une interaction auto-créatrice. Dès lors, la question délicate est de savoir comment se déploie le dialogue.

Prendre au sérieıx le caractère éminemment nrocessuel des dialoogues exclut toute 
appréhension structurelle ou procédurale qui interviendrait post festum. Pour autant, il ne faudrait pas croire que ce procès dialogique se déroule de façon totalement aléatoire. En tant qu'activité coopérative entre agents rationnels, il est contrôlé et guidé par un modèle projectif qui fournit aux interlocuteurs un cadre générique permettant de canaliser leurs attentes et d'évaluer leurs réactions mutuelles. Ainsi, à chaque grand type d'interaction correspond un modèle projectif ${ }^{22}$. L'exemple le plus simple parce que celui où l'enjeu, les buts, les rôles sont nettement définis est le modèle projectif d'un dialogue informatif élémentaire. Dans [Vernant, 1992], nous en proposions une schématisation dont nous rappellerons ici seulement les grandes lignes.

Tout dialogue informatif élémentaire (consultation d'une base de connaissances ou interrogation d'un service de renseignement) constitue une activité conjointe qui suppose que les agents aient non pas le même but, mais des buts complémentaires, croisés (offre/demande), qui autorisent l'échange d'information. Tout dialogue informatif se fonde sur la différenciation initiale des interlocuteurs. Du point de vue informatif, leurs rôles sont strictement différenciés et asymétriques : le locuteur désire une information que l'allocutaire peut et veut fournir. L'un est demandeur, l'autre répondant. Cette polarité informationnelle conditionne la possibilité même de ce type de dialogue. On notera de plus, qu'au regard de la finalité informationnelle, le répondant possède un primat incontestable : le demandeur interroge le répondant comme on consultait l'oracle. Le but du dialogue est alors de réduire l'écart cognitif initial entre demandeur et répondant, d'instaurer une convergence prédicative et référentielle telle qu'in fine la demande initiale d'information soit satisfaite. Graphiquement, nous avons proposé de représenter cette convergence par une construction en $\mathrm{V}$, ou en entonnoir, renversés. À partir de la différence informationnelle initiale, les champs sémantique et référentiel doivent idéalement se réduire progressivement pour parvenir à l'accord final impliquant l'atteinte du but : le partage de l'information. 


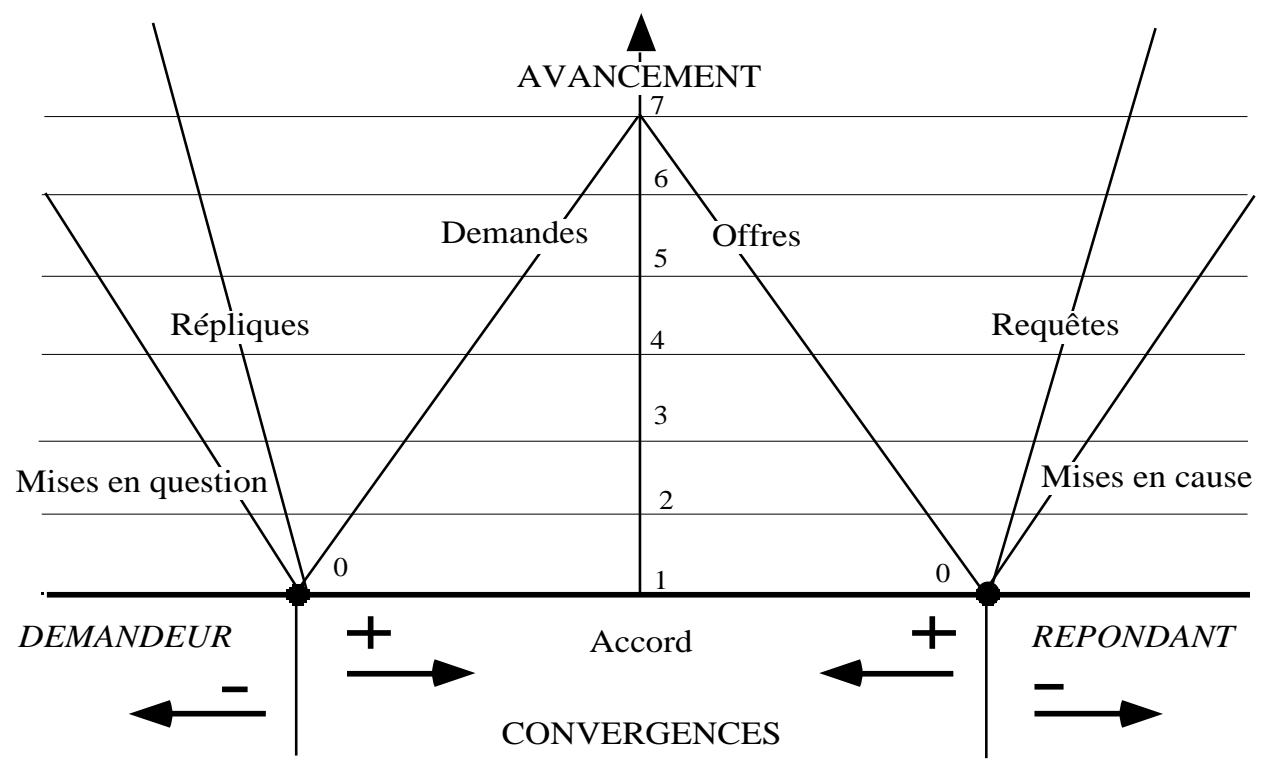

DIVERGENCES

DIVERGENCES

\section{MODÈLE PROJECTIF DU DIALOGUE INFORMATIF}

Le schéma minimal d'un tel dialogue se réduit à trois interventions :

$1^{\circ}$ - le demandeur énonce sa demande d'information : «Quelle heure est-il ? »,

$2^{\circ}$ - le répondant fournit l'information souhaitée : «Il est 17 h 40 »;

$3^{\circ}-$ le demandeur, après évaluation, accepte l'information : «Merci ».

Il est clair toutefois que cette convergence informationnelle ne peut généralement être atteinte qu'après un procès plus complexe au cours duquel la demande initiale se décline en demandes annexes et les offres d'information se précisent. C'est pourquoi on distingue à gauche pour le demandeur une ligne des demandes et à droite pour le répondant une ligne des offres.

De plus, la convergence est généralement rompue provisoirement par des phénomènes de divergence qui se caractérisent par une inversion des rôles dialogiques. Si, par exemple, le répondant ne comprend pas un terme employé par le demandeur, il va non plus répondre assertivement, mais faire une requête en posant une question du genre : «Que voulez-vous dire par ... ? » et ainsi inaugurer une séquence métadiscursive. Réciproquement, le demandeur pourra non plus questionner, mais répliquer. Enfin, dans les cas les plus graves de divergence, ce sont les présupposés communs au dialogue qui seront mis en cause par le répondant ou mis en question par le questionneur, cf. [Vernant, 1997, 107-125].

Un tel modèle projectif assure l'intelligibilité du déroulement du dialogue. Il permet d'assigner une fonction dialogique aux interactes émis. On a vu précédemment qu'un acte de discours pris dans l'interaction pouvait prendre diverses forces illocutoires. Mais bien plus, des interactes de même force illocutoire peuvent prendre diffé-

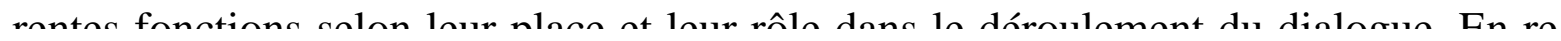


situant les interactes dans le cadre plus large d'un modèle projectif d'interaction, il devient alors possible de définir pragmatiquement les fonctions dialogiques effectives qu'ils assurent. Apparaît alors la foncière multifonctionnalité des actes de discours. Ainsi, pour n'examiner qu'un seul type de force, des actes auxquels on pourra attribuer une force assertive prendront selon le contexte dialogique des fonctions différentes. Un même assertif, dans des dialogues différents, prononcé par des interlocuteurs différents ou par le même interlocuteur à des moments différents, etc., prendra des fonctions dialogiques différentes. Examinons l'échange téléphonique suivant entre un usager et un agent du service de renseignements SNCF :

1 - Allô,

2 - Je suis à Grenoble et je souhaite aller demain à Paris,

3 - Le premier train part à $7 \mathrm{~h} 10$,

4 - Ah, bon, d'accord. Et pour le retour jeudi. Je dois être à Grenoble à 13 heures,

5 - Vous avez le $9 \mathrm{~h} 48$ qui arrive à 12 h 54 . Vous serez juste à l'heure,

6 - Non, il risque d'avoir du retard comme la dernière fois,

7 - Alors, prenez le 8 h 24 qui arrive à 11 h 26 ,

8 - Mais, j'y pense, on annonce un mouvement de grève en fin de semaine,

9 - Je ne suis pas au courant,

10 - je vais me renseigner, merci.

Selon notre modélisation, on peut schématiser ce dialogue ainsi :

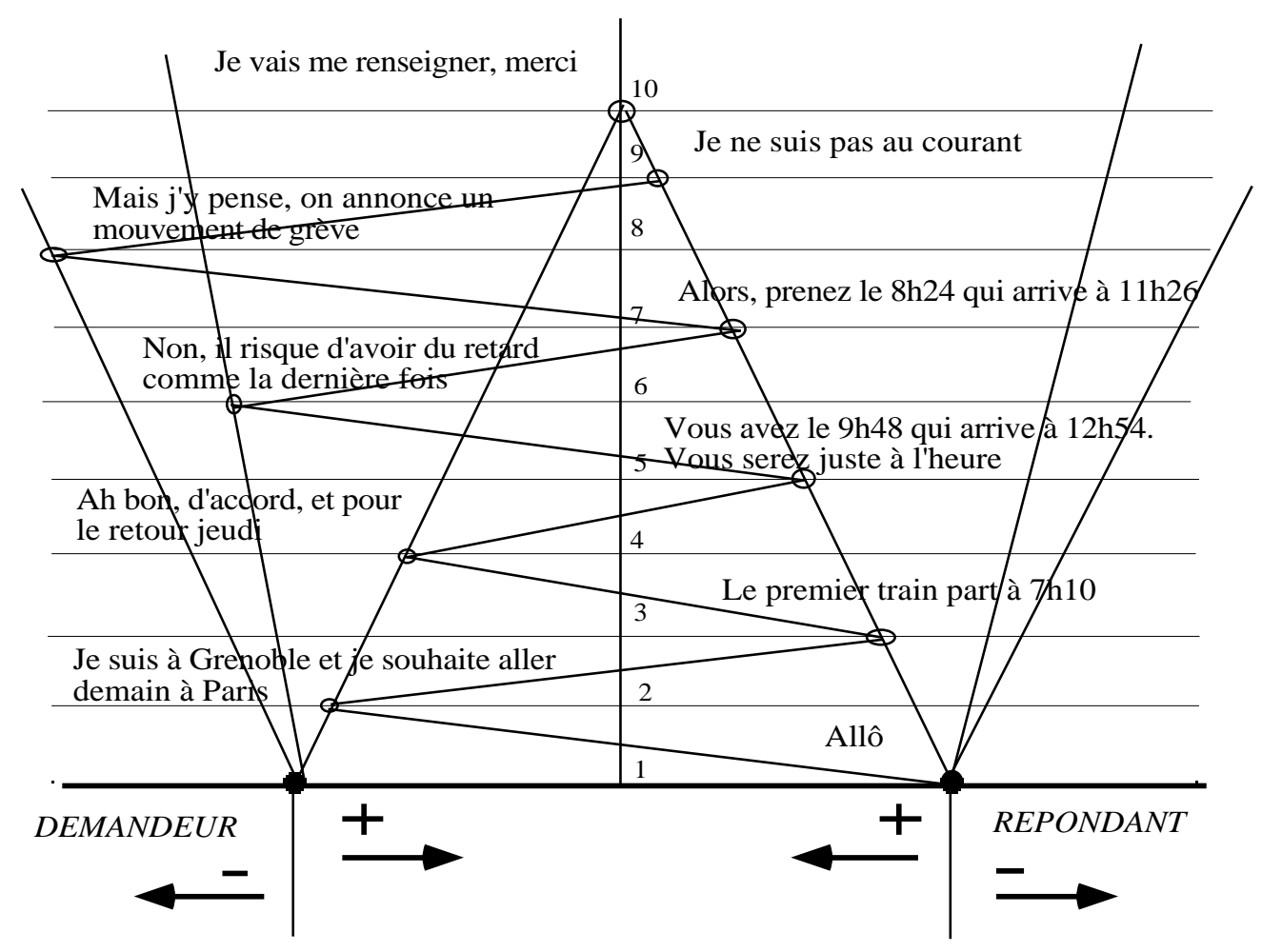


La première partie de l'énonciation 2 «Je suis à Grenoble » prendra fonction de proposition : information initiale qui contribue à cerner d'emblée le problème. La seconde partie « je souhaite aller demain à Paris » peut abstraitement être considérée comme un expressif explicitant un souhait du locuteur. Manifestement, il complète la formulation du problème. Étant donné le contexte précis de l'interaction, cet acte sera immédiatement interprété par le répondant comme une demande d'information, une question : «Comment me rendre demain par le train à Paris ? ». Cette question produira une assertion 3 comme réponse : «Le premier train part à 7 h 10 ». Le demandeur pourra dire en $4:$ «Ah, bon, d'accord ». On aura alors une nouvelle assertion ayant fonction d'évaluation exprimant en l'occurrence un assentiment (cela aurait pu être un dissentiment qui aurait relancé l'interrogation). Proposition, réponse et assentiment constituent ainsi diverses fonctions que peut prendre l'assertion dans un dialogue qui converge. À un stade de moindre convergence, on aurait pu ajouter la fonction métacommunicationnelle d'une assertion qui vaut reformulation d'un propos antérieur du locuteur pour assurer sa compréhension par l'allocutaire. Par exemple, l'opérateur aurait pu confirmer : «Oui, il y a un train pour Paris à 7 h 10 du matin ».

Mais en cas de divergence marquée, une assertion peut acquérir valeur de réplique ou, plus grave, de mise en cause. La seconde partie de l'énonciation 4 constitue une demande d'information à laquelle il est répondu pertinemment en 5 . Le demandeur aurait dû normalement clore l'entretien en validant la réponse apportée ou bien relancer le dialogue en posant une nouvelle question. Or, il ne fait rien de tout cela et émet en 6 une assertion qui prend valeur de réplique. On a là une première divergence que l'interlocuteur, délibérément ou non, minimise en transformant en simple question du type « À quelle heure part le train suivant ? ». Il y répond en 7. Le demandeur ne discute pas cette réinterprétation ni ne la valide parce qu'il aggrave la divergence en mettant en cause cette fois un présupposé de l'interaction entre usager et agent SNCF par 8 : «Mais, j'y pense, on annonce un mouvement de grève en fin de semaine ». On a là une mise en cause qui menace directement l'interaction dans la mesure où une demande de renseignement SNCF présuppose généralement une situation normale. Sauf à rompre le dialogue, le répondant doit alors changer de registre, abandonner la consultation des horaires pour entrer dans des considérations socio-politiques. $\mathrm{Ce}$ qu'il ne fait pas, fidèle à sa stratégie consistant à réduire les divergences. Mais son « Je ne suis pas au courant » en 9 , sincère ou feint, est non pertinent, c'est pourquoi le demandeur rompt le dialogue, non sans toutefois valider l'apport d'information par l'énonciation $10:$ : Je vais me renseigner, merci ».

Comme le montre cet échange ${ }^{23}$, dans sa réalité dialogique, l'assertion peut ainsi

23. Plausible, un tel dialogue est cependant fictif : il a pour seul objet pédagogique de fournir un exemple 
paraître sous forme de proposition, réponse, as(dis)sentiment, reformulation, réplique ou mise en cause. Il en va de même, mutatis mutandis, pour tous les autres types d'acte.

\section{4 - Sa finalité transactionnelle}

À cette définition du dialogue, il convient d'ajouter un dernier trait, capital : le caractère foncièrement hétéronome de l'interaction langagière. In fine, on ne parle jamais pour parler, mais pour agir : pour construire un monde avec, pour, par, ou contre l'autre. D'où la nécessité d'adjoindre une dimension transactionnelle de l'analyse qui croise le procès intersubjectif de co-constitution des interlocuteurs avec celui intramondain de co-construction de mondes. Ainsi, c'est à ce niveau ultime de la finalité actionnelle que doivent désormais être repensées les antiques questions de la signification et de la vérité. En résulte par exemple, une conception résolument non-représentationnelle du sens et non-correspondantiste de la vérité.

Contre les philosophes du dialogue qui reproduisent le postulat d'immanence saussurien au niveau de la pratique discursive en maintenant l'illusion d'une autonomie de l'agir communicationnel et en imposant une clôture logocentrique de toute appréhension du sens, nous soutenons le caractère foncièrement hétéronome de toute forme d'échange langagier. L'analyse pragmatique des échanges langagiers ne saurait se suffire à elle-même. L'interaction communicationnelle trouve son sens et sa finalité dans une transaction de nature non langagière. Une telle transaction met en jeu aussi bien les relations intersubjectives dans leur dimensions psychologique et sociale que les rapports des acteurs aux mondes qu'ils construisent ensemble. On parle avec autrui pour agir avec lui ou le convaincre d'agir sur un monde momentanément partagé. L'interaction qui instaure un échange langagier entre interlocuteurs en permettant notamment de s'accorder sur une image du monde, trouve sa finalité transactionnelle dans les relations que tissent les sujets ainsi que dans leurs interventions conjointes sur un monde qu'ils contribuent à construire. Activité essentielle, l'usage du langage permet la recollection et l'analyse des faits, la réflexion sur les buts, l'évaluation des stratégies, la décision d'action. Comme tel, il a valeur de détour langagier subordonné à des enjeux et des finalités non langagières. Dans cette perspective transactionnelle, le dialogue constitue une pratique langagière de coopération visant la constitution d'information, l'accord sur les fins et les moyens, la réalisation des actions. En Intelligence artificielle, cette distinction et articulation des niveaux est patente. On construit une application informatique pour réaliser une tâche spécifiée et l'on prévoit la possibilité d'une interface pour assurer l'interaction entre l'utilisateur et la machine.

De façon générale, on peut illustrer l'articulation des niveaux inter- et transactionnel par le schéma suivant où les arcs figurent les interactions langagières et les seg- 


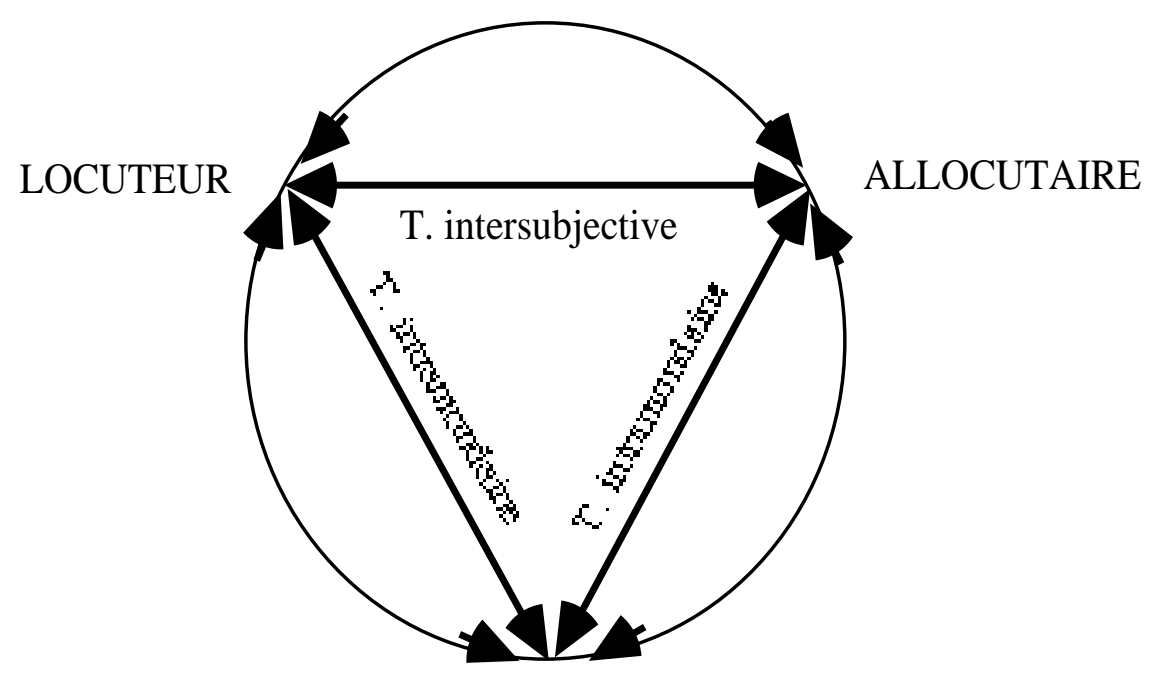

Image du / MONDE

Ainsi, la transaction s'inscrit au cœur du dispositif interactionnel. Bien sûr, le langage contribue à constituer et à réfléchir les rapports intersubjectifs et intramondains, mais de tels rapports s'incarnent dans des transactions effectives extra-langagières. Ainsi prendra-t-on garde au fait que les actes de discours sont foncièrement pluridimensionnels. Ils possèdent naturellement une dimension interactionnelle comme éléments d'un échange conversationnel, mais aussi, pour une part, ils contribuent à la régulation transactionnelle des relations intersubjectives et de la construction d'une image du monde. On retrouve ici, déployée sur les rapports intersubjectifs et intramondains, la dimension perlocutoire d'Austin. « Ferme la porte » traduit bien un désir du locuteur, mais surtout cet acte de discours intime à l'allocutaire l'ordre d'agir sur la situation partagée. Le choix d'un ordre direct plutôt qu'une forme affaiblie : «Auriez-vous l'obligeance de fermer la porte » ou indirecte : «Vous serait-il possible de fermer la porte ? » prend en compte les places respectives des interlocuteurs, le caractère plus ou moins hiérarchique et contraignant de leurs rapports interpersonnels ${ }^{24}$.

\section{$\underline{\text { A-Transactions intersubjectives }}$}

Un premier aspect de la transaction porte sur les relations interpersonnelles. L'objet premier du dialogue est celui de la transformation mutuelle des états mentaux des interlocuteurs : de leurs croyances et connaissances (dimension cognitive), de leurs désirs et intentions (dimension volitive), de leurs valeurs et interdits (dimension axiolo-

24. Pour tenir compte de cette pluridimensionnalité, on fera appel à un contexte interactionnel comprenant le cotexte dialogique et les rôles des interlocuteurs, leurs croyances et connaissances, leurs présupposés communs,... ainsi qu'un contexte transactionnel comprenant les circonstances (lieu, temps, acteurs, problème) 
gique) ainsi que de leurs sentiments et émotions (dimension affective) ${ }^{25}$. Sont en jeu les composants psychologique et social des personnes. En plus des informations contenues dans l'échange verbal, les éléments prosodiques du dialogue oral (ton, rythme, etc.), mais aussi les regards et mimiques, les gestes et postures fournissent des indices de ces transactions interpersonnelles. On sait depuis Platon que l'exercice philosophique comme questionnement engage un désir de vérité qui passe par un désir de l'autre. Plus généralement, toute érotétique suppose, sous une forme ou une autre, une érotique. Sont en cause les places et faces, les statuts et images que les interlocuteurs constituent dans et par l'interaction communicationnelle. C'est ici, par exemple, qu'interviennent les règles de politesse : le « Soyez poli » que [Grice, 1975, 62] excluait de ses maximes conversationnelles. Une telle règle est humainement aussi importante que le «Soyez pertinent », simplement elle ne relève pas du même niveau $^{26}$. On sait de plus que bien souvent les contraintes techniques imposées par les maximes conversationnelles se trouvent subordonnées aux règles de politesse : il est des cas où, par politesse, pour ne pas « froisser » l'allocutaire, il importe de ne pas être clair ou de ne pas être tout à fait sincère. On pourrait ainsi, selon les types d'interaction, les statuts des interlocuteurs, les types de culture, édicter des règles gouvernant les transactions intersubjectives. Une négociation commerciale entre deux industriels à Paris diffère toto caelo des palabres préludant à une vente de terrain entre deux paysans de la Creuse !

\section{$\underline{B \text { - Les transactions intramondaines }}$}

Venons-en maintenant à la dimension intramondaine de la transaction. Nous soutenons que tout dialogue est soumis à des enjeux transactionnels qui lui assignent sens et finalité. Naturellement, ce principe doit être modulé selon les types de dialogue en cause. La conversation à bâtons rompus, les bavardages de salon, les échanges entre voisins dans l'ascenseur, ont une finalité principalement - voire exclusivement - phatique, intersubjective et non intramondaine. Il s'agit de prendre langue avec le voisin, le convive, pour le plaisir de l'échange, ou pour «alimenter la conversation » et non pour traiter une question, résoudre un problème, réaliser une tâche. À l'autre extrême, les débats philosophiques, les discussions entre experts en art, les controverses scientifiques peuvent se présenter comme des pratiques dialogiques supérieures, autonomes, i.e. libérées des viles contraintes transactionnelles. Ces formes hautement culturelles de dialogues viseraient une connaissance du Vrai et du Beau, une pure contemplation des Idées. En fait, elles s'avèrent des détours langagiers sophistiqués tributai-

25. Sous peine d'incohérence, ces états mentaux, par-delà leur expression représentationnelle, doivent être interprétés en termes actionnels, pragmatiques. Par exemple, les croyances des interlocuteurs relèvent de dispositions à agir, cf. [Peirce, 1960, 5.400] : " Toute la fonction de la pensée est de créer des habitudes d'action » et les valeurs s'interprètent en termes de raisons d'agir. 
res in fine d'enjeux transactionnels prosaïques. L'idéal de l'art pour l'art ou du savoir comme contemplation désintéressée a fait long feu !

À mille lieues tant de ces formes exclusivement phatiques que des constructions verbales les plus éthérées, le dialogue informatif entre l'Homme et le système informatique se trouve immédiatement tributaire d'une finalité intramondaine. L'action dialogique, qui naît d'un manque d'information, a valeur de quête de savoir en vue d'une action non langagière. Ainsi, l'interrogation d'une base de connaissances constitue manifestement une activité hétéronome en ce que sa finalité est subordonnée à un but extrinsèque de nature non discursive. Sauf exception, on n'interroge pas l'horloge parlante uniquement pour connaître l'heure, mais en vue de programmer une action. L'activité dialogique s'inscrit explicitement dans une chaîne d'actions non langagières. Dès lors, l'action informative est déterminée par un contexte actionnel plus général. L'intention du locuteur est un désir de savoir pour agir ${ }^{27}$. Ceci s'exprime techniquement par le fait que l'on a affaire à un dialogue « orienté par la tâche » [Pierrel, 1987, 160] utilisant un « langage opératif » dont la syntaxe et surtout la sémantique (pauvre) sont directement déterminées par le but transactionnel [Falzon, 1989]. On mesure ici l'importance d'une réinterprétation des actes de discours produits au cours du dialogue en termes actionnels plus généraux. Car ces actions langagières ne prennent sens qu'à être référées à des actions non langagières passées et futures. Le modèle de la tâche a pour objet de préciser ces contraintes et de fournir les informations nécessaires aux inférences produites par les interlocuteurs. Ainsi le modèle de la tâche apparait-il comme l'explicitation informatique en termes de planification ou de scénarios d'une partie très spécifiée et limitée du champ d'action qui conditionne l'échange dialogique. Le caractère artefactuel du dialogue personnemachine provient précisément du fait que la tâche étant clairement circonscrite et précisément délimitée, il est relativement possible de maîtriser l'ensemble, potentiellement infini, des connaissances impliquées et des savoir-faire requis, cf. [Vernant, 2003a].

De façon générale, les interactions langagières doivent être situées dans cette perspective transactionnelle. Les actes de discours des interlocuteurs prennent sens en fonction de leurs intentions, buts et plans d'action sur un monde qu'ils co-construisent ensemble. Confrontés à la même situation et au même problème, les co-agents voient s'ouvrir des espaces d'intervention qui délimitent leurs intentions ${ }^{28}$. Si l'accord interactionnel est rendu possible par le partage de modèles dialogiques, l'accord transac-

27. Il est même des cas fréquents où transactions extra-langagières et interactions langagières sont étroitement imbriquées, tel lors de l'achat de places de cinéma : «- Deux places pour : ... en tendant un billet de vingt Euros, — Douze Euros, en rendant la monnaie, — Merci ».

${ }^{28}$ Cf. [Wittgenstein, 1969, § 337] : «L'intention est inhérente à la situation, aux coutumes et aux 
tionnel l'est par le partage d'une même «forme de vie ». Ces formes de vie sont techniquement et socialement déterminées. Elles varient dans l'espace et dans le temps. Sous une forme ponctuelle, elles peuvent s'incarner en des scénarios stéréotypés qui fournissent un cadre au déroulement des actions et un sens à l'interaction langagière ${ }^{29}$. De façon plus globale, est en jeu ce que Wittgenstein appelle « image du monde ${ }^{30}$ qui n'est en rien une représentation conceptuelle de ce monde, mais l'arrière-plan actionnel qui, commun aux co-agents, autorise et oriente leur coopération effective ${ }^{31}$. On atteint là le soubassement proprement culturel et anthropologique du sens des actions humaines. Dès lors, le monde, contrairement à la conception qui gouverne l'attitude naturelle comme de nombreuses analyses philosophiques et techniques, s'avère non une réalité ultime, objective, donnée immédiatement, mais une réalisation collective, produit des interactions langagières comme des transactions non langagières. Il en résulte que chaque monde est provisoire et qu'existe une pluralité de mondes, cf. [Goodman, 1978]. Le monde de la vie quotidienne qui est en question dans la plupart de nos conversations a peu de rapports avec le monde des physiciens qui est en jeu dans les controverses en théorie quantique ${ }^{32}$, etc. On comprend alors pourquoi la vérité ne peut plus se définir en termes de correspondance du discours au réel, mais doit faire l'objet d'une conceptualisation proprement praxéologique.

Le dessin de la destinée humaine se tisse sur une toile dont la chaîne est constituée d'une multitude de dires, interactions assurant la communication intersubjective, et dont la trame est composée d'innombrables faires, transactions produisant effectivement des transformations sur nos mondes.

29. Que l'on pense par exemple au scénario qui conditionne les transactions et interactions dans un restaurant. On notera qu'au niveau élémentaire les dispositifs d'action, généralement corporels, n'ont pas à faire l'objet d'une décision. Si je décide de répondre au sommelier qui me somme (en vertu des pouvoirs que lui confère le scénario) de goûter le vin, je ne décide pas de tendre le bras vers le verre, le saisir, le porter à mes lèvres, de boire une gorgée, etc. Sur la question des scénarios en I.A, cf. [Schank \& Abelson, 1977].

30 . « Mais cette image du monde, je ne l'ai pas parce que je me suis convaincu de sa rectitude; ni non plus parce que je suis convaincu de sa rectitude. Non, elle est l'arrière-plan dont j'ai hérité sur le fond duquel je distingue entre vrai et faux », [Wittgenstein, 1969, § 94].

31. Selon [Searle, 1983, ch. 5], qui reprend la notion wittgensteinienne, cet arrière-plan serait «préreprésentationnel ». Comme Searle développe une conception strictement représentationnelle du sens, on voit mal comment peut être exploité cet arrière-plan. Ce hiatus disparaît si on adopte pour le sens, les croyances, etc. une conception actionnelle.

32. On peut développer une conception dialogique des sciences en insistant sur le rôle de la théorie et des controverses intrathéoriques. Mais il convient de croiser cette dimension interactionnelle avec les contraintes transactionnelles : le rôle des protocoles expérimentaux et des techniques de «fabrique des phénomènes », les déterminations socio-historiques qui commandent les enjeux et systèmes de valeurs, les engagements économiques et les choix de politiques de recherche, voire même, lorsque l'analyse atteint un certain degré de finesse, 


\section{Conclusion}

Depuis Frege, le paradigme actionnel s'est considérablement développé et s'est finalement imposé comme moyen de rendre compte de la complexité des phénomènes communicationnels humains. Tout n'est pas pour autant résolu. Trois pistes de recherche me paraissent s'imposer :

$1^{\circ}$ - approfondir les présupposés philosophiques d'un tel paradigme, par exemple développer une conception résolument non représentationnelle du sens et non correspondantiste de la vérité.

$2^{\circ}$ - préciser les analyses de l'interaction et de ses contraintes transactionnelles et en tenter la formalisation sans succomber aux dérives logicistes.

$3^{\circ}$ - enfin et surtout, in cauda venenum, élaborer une praxéologie ou théorie générale de l'action qui fournisse les concepts opératoires applicables à toute forme d'action, communicationnelle ou non.

Un tel programme déborde largement le champ de la seule philosophie du langage et requiert manifestement un approche résolument interdisciplinaire dont notre présente rencontre peut utilement tracer les jalons.

\section{RÉFÉRENCES BIBLIOGRAPHIQUES}

(Les dates sont celles des éditions originales, les paginations renvoient aux traductions françaises quand elle existent)

Austin John, 1962, How to do Things with Words, Oxford U.P. ; tr. fr. G. Lane, Quand dire, c'est faire, Paris, Seuil, 1970.

ARMENGAUd Françoise, 1985, La Pragmatique, Paris, PUF.

Clancey William J., 1993, «Situated Action : A Neuropsychological Interprétation. Response to Vera and Simon », Cognitive Science, vol. 17, ${ }^{\circ}{ }^{1}$, Norwood, New Jersey, Ablex Publishing Corporation, p. 87-116.

COLINEAU Nathalie, 1997, Étude des marqueurs discursifs dans le dialogue finalisé, thèse, déc. 1997, CliPS-IMAG, Grenoble. 
DAVAl René, 1997, Moore et la philosophie analytique, Paris, PUF, Coll. Philosophies.

DAVIDSON Donald, Inquiries into Truth \& Interprétation, Oxford U.P., New-York, 1984 ; tr. P. Engel, Enquêtes sur la vérité et l'interprétation, Nîmes, Éd. J. Chambon. 1993.

ESPINAS Alfred, 1897, Les Origines de la technologie, études sociologiques, Paris, Alcan.

FALZON Pierre, 1989, Ergonomie cognitive du dialogue, P.U. Grenoble.

FERBER Jacques, 1995, Les Systèmes multi-agents, Paris, Interéditions.

FREGE Gottlob, 1892, «Sinn und Bedeutung », Zeitschrift für Philosophie und philosophische Kritik, 100 ; tr. fr. par Cl. Imbert, «Sens et dénotation », Écrits logiques et philosophiques, Paris, Seuil, 1971, p. 102-126.

FREGE Gottlob, 1918, « Die Verneinung », Beiträge zur Philosophie des deutschen Idealismus, 1, n³-4, 1918-1919, p. 143-157 ; tr. fr. par Cl. Imbert, « La négation », Écrits logiques et philosophiques, Paris, Seuil, 1971, p. 195-213.

Goodman Nelson, 1978, Ways of Worldmaking, Hackette Publishing Company ; tr. fr. par M.-D. Popelard, Manières de faire des mondes, Nîmes, Ed. J. Chambon.

BROCK Jarrett E., 1981, « An Introduction to Peirce's Theory of Speech Acts », Transaction of the C.S. Peirce Society, 1981, XVII, n²4, p. 319-326.

Grice PAUL, 1975, «Logic and Conversation » in Syntax and Semantics 3 : Speech Acts, Coles \& Morgan, New-York, Academic Press, p. 41-58, ; tr. fr. par F. Berthet \& M. Bozon, «Logique et conversation », Communications, Paris, Seuil, juin 1979, $\mathrm{n}^{\circ}$ spécial 30, p. 57-72.

KERBRAT-ORECCHIONI Catherine, 1996, «Variations culturelles et universaux dans le fonctionnement de la politesse linguistique », Le dialogique, sur les formes philosophiques, littéraires, linguistiques et cognitives du dialogue, Berne, Peter Lang Ed., coll. Sciences pour la communication.

MeYer Michel, 1986, De la problématologie, Paris, Le livre de poche.

Moore George, E., 1942, « A Reply to my Critics », in The Philosophy of Moore, P. A. Schilpp (ed.), Open Court, p. 542-3. 
NeUBERG Marc, 1991, Théorie de l'action, textes majeurs de la philosophie de l'action, Bruxelles, Mardaga.

PeIrce Charles Sanders, 1960, Collected Papers, C. Hartshorne \& P. Weiss (éds.), Cambridge, Mass., Harvard U.P.

PIERREL Jean-Marie, 1987, Dialogue oral Homme-Machine, Paris, Hermès.

SCHANK R.C. \& ABELSON R.P., 1977, Scripts, Plans, Goals and Understanding : an Inquiry into Human Knowledge Structures, Erlbaum, Hillsdale, New-Jersey.

SEARLE John, 1983, Intentionality, an Essay in the Philosophy of Mind, Cambridge U. P. ; tr. fr. par Cl. Pichevin, L'Intentionalité [sic], essai de philosophie des états mentaux, Paris, Éd. de Minuit, 1985.

SEARLE John, 1968, « Austin on Locutionary and Illocutionary Acts », The Philosophical Review, 77, n 4, p. 405-424.

Suchman Lucy, 1987, Plans and Situated Action : The Problem of Human-Machine Communication, New York, Cambridge University Press.

TROGNON Alain et BRASSAC Christian, 1992, « Théorie des actes de langage et analyse des conversations », Cahiers de linguistique française, $\mathrm{n}^{\circ}$ 13, Genève.

VANDERVEKEN Daniel, 1999, «La structure logique des dialogues intelligents », in B. Moulin, S. Delisle \& B. Chaib-draa dirs., Analyse et simulation des conversations, Coll. Informatique, L'Interdisciplinaire, Lyon.

Vera Alonso H. \& SimON Herbert-A., 1993, « Situated Action : A Symbolic Interprétation » Cognitive Science, vol. 17, ${ }^{\circ} 1$, Norwood, New Jersey, Ablex Publishing Corporation, p. 7-48.

VARELA Francisco, 1993, «L'enaction : cognition incarnée », in F.Varela, E. Thompson, E. Rosch, L'Inscription corporelle de l'esprit, Sciences cognitives et expérience humaine, Paris, Seuil, p. 207-248.

VERNANT Denis, 1992, «Approche actionnelle et modèle projectif du dialogue informatif », in Du Dialogue, D. Vernant (éd.), Recherches sur la philosophe et le langage, $\mathrm{n}^{\circ} 14$, Paris, Vrin.

Vernant Denis, 1997, Du Discours à l'action, études pragmatiques, Paris, PUF.

VERNANT Denis, 1999, «Analyses sémantique, pragmatique et praxéologique des phénomènes communicationnels », in Analyse et simulation des conversations. De la 
théorie des actes de discours aux systèmes multi-agents, B. Moulin \& B. Chaib-draa, éds., L'Interdisciplinaire, Lyon.

VERNANT Denis, 2003a, «Communication interpersonnelle \& communication personnes/systèmes » in Communication entre personnes et systèmes informationnels, B. Miège, Paris éd., Hermès.

VERnANT Denis, 2003b, «The Limits of a Logical Treatment of Assertion ». D.Vanderveken ed., Logic, Thought and Action, Netherlands, Dordrecht, Kluwer.

WitTGENSTEIN Ludwig, 1953, Philosophische Untersuchungen, Oxford, Blackwell ;

tr. fr. par P. Klossowski, Investigations philosophiques, Paris, Gallimard, Tel, 1989.

WitTGENSTEIN Ludwig, 1969, Über Gewissheit, Oxford, Blackwell, 1969 ; tr. fr. par J. Fauve, De la Certitude, Paris, Gallimard, Coll. Tel, 1976.

Denis.Vernant@upmf-grenoble.fr http://www.upmf-grenoble.fr/SH/Perso/DenisVernant/presentation.html

Denis Vernant est professeur de philosophie à l'Université Pierre Mendès France de Grenoble où il dirige le groupe de recherche Philosophie, langages \& Cognition. Il enseigne la logique et la philosophie de la logique, la pragmatique et la praxéologie. Il a publié notamment : Introduction à la philosophie de la logique, Bruxelles, Mardaga, 1986 ; La Philosophie mathématique de B. Russell, Paris, Vrin, 1993 ; Du Discours à l'action, études pragmatiques, Paris, PUF, 1997 ; Introduction à la logique standard, Paris, Flammarion, 2001 et Bertrand Russell, Paris, Flammarion, 2003. 